\title{
Symptomatic treatments of $N$-methyl-D-aspartate receptor encephalitis
}

\author{
Soon-Tae Lee \\ Department of Neurology, Seoul National University Hospital, Seoul, Korea
}

$\mathrm{N}$-methyl-D-aspartate receptor (NMDAR) encephalitis presents with multiple symptoms including memory loss, seizure, psychosis, aphasia, altered mentality, dyskinesia, autonomic dysfunction, and central hypoventilation. While immunotherapy protocols are improving, morbidity and mortality in the disease largely depend on supportive care to control intractable symptoms. However, no prospective or controlled trials have been conducted on immunotherapy or supportive care principles in the disease. Thus, this study discusses and shares experience and ideas for symptomatic care of NMDAR encephalitis.

Keywords: N-methyl-D-aspartate receptor, Encephalitis, Symptomatic, Treatment

\section{Introduction}

$N$-methyl-D-aspartate receptor (NMDAR) encephalitis is the most common and serious form of autoimmune encephalitis. It is caused by autoantibody against NR1 subunit of the NMDAR receptor. Key symptoms include memory loss, psychosis, seizure, aphasia, altered mentality, dyskinesia, autonomic dysfunction, and central hypoventilation [1]. Immunotherapy is the main treatment and includes steroid, immunoglobulin, rituximab, tocilizumab, and/or cyclophosphamide, which are frequently combined to enhance outcomes. Although the final outcome has been improved after the application of novel immunotherapies, there still exist a lot of mortality and permanent morbidity by the disease. When treated for up to 24 months, $25 \%$ of patients remained disabled in 3 to 6 categories of the modified Rankin Score [1]. Hence, long-term integrated supportive care is essential until the full effect of immunotherapy can ameliorate disease symptoms.

This paper summarizes the details of supportive care for disabling symptoms of NMDAR encephalitis based on reported evidences and personal experience.

\section{Memory Loss}

Because NMDAR mediates long-term potentiation of memory [2], memory loss is the early and key symptom in NMDAR encephalitis. However, currently no symptomatic treatment is available to enhance memory function in this disease. EphB2 receptors form a complex with NMDAR, and anti-NMDAR antibodies disrupt the cross-talk between NMDAR and EphB2 receptors [3]. Moreover, ephrin-B2 (the ligand of the EphB2 receptor) prevents intracellular internalization of NMDAR by disease antibodies [4]. Accordingly, some chemicals modulating the EphB2 receptor could affect NMDAR encephalitis symptoms; however, no clinically usable drugs have been approved for this purpose. Because memory loss is largely reversible after remission of the disease, education regarding the disease course and psychological support for family members are necessary.

\section{Psychosis}

Psychosis and behavioral problems not only increase caregiver burden but also can result in self-injury. To control psychosis, antipsychotics such as dopaminergic blockers and anxiolytics

Received: November 4, 2020 Revised: November 30, 2020 Accepted: December 1, 2020

Correspondence: Soon-Tae Lee

Department of Neurology, Seoul National University Hospital, 101 Daehak-ro, Jongno-gu, Seoul 03080, Korea

E-mail: staelee@snu.ac.kr

ORCID: https://orcid.org/0000-0003-4767-7564

Copyright (C) 2021 by The Korean Encephalitis and Neuroinflammation Society

This is an open access article distributed under the terms of the Creative Commons Attribution Non-Commercial License (http://creativecommons.org/licenses/by-nc/4.0/) which permits unrestricted non-commercial use, distribution, and reproduction in any medium, provided the original work is properly cited. 
such as benzodiazepine can be prescribed. Because the pattern of psychosis is variable [5], control of symptoms depends on individual phenotypes. While psychiatric symptoms that emerge in the early disease phase can be partially controlled by antipsychotics, close monitoring is necessary because antipsychotics can aggravate rhabdomyolysis or induce neuroleptic malignant syndrome, especially in patients with dyskinesia [6]. Atypical antipsychotics can reduce extrapyramidal side effects, although further studies are needed. Similar to other symptoms of the disease, immunotherapy is the main tool to control psychosis. Meanwhile, psychosis is often re-aggravated during the later stages of the disease, and re-introduction of antipsychotics is sometimes necessary. Hyperactive forms of psychosis can reemerge, such as anxiety, anger, or disinhibition, usually accompanied by improvements in memory function.

\section{Dyskinesia}

Dyskinesia is one of the most troublesome symptoms of the disease. Down-regulated NMDAR expression causes dopaminergic overactivation in the globus pallidus and might cause hyperkinetic movement disorders [7]. Severe dyskinesia causes self-injury, rhabdomyolysis, and renal failure [6]. In intensive care units, anesthetics or neuromuscular blockers are necessary in severe cases. However, when dyskinesia continues for several months, prolonged use of these agents is not clinically feasible due to other systemic complications.

Recently, I have shown that mega-dose enteral diazepam (ranging from 6 to $180 \mathrm{mg}$ ) can control dyskinesia effectively [8]. Previously, diazepam has demonstrated muscle-relaxant properties in neuroleptic malignant syndrome and tetanus $[9,10]$. In the severe form of dyskinesia resulting from NMDAR encephalitis, mega-dose diazepam decreased the severity of dyskinesia to tolerable levels and no patients had serious adverse events except mild sedation [8]. By using mega-dose diazepam, I could stop anesthetics or neuromuscular blockers in many patients and the effect was durable for the remaining immunotherapy period. I usually start diazepam at a medium dose (3-5 mg, three or four times daily) and then double the dose every several days depending on response, up to a maximum of $180 \mathrm{mg}$ daily. Mega-dose enteral diazepam could be an effective treatment option for controlling dyskinesia in NMDAR encephalitis.

\section{Seizure}

The management of epilepsy in NMDAR encephalitis is in line with the management of autoimmune epilepsy, the clinical ap- proach of which is described in a previous consensus [11]. In terms of antiepileptic drug (AED) selection, selection depends on clinician preference, seizure semiology, related medical conditions, and side effect profiles. Drugs with fewer side effects, no drug-drug interactions and that are fast loading are preferred, and next-generation AEDs such as levetiracetam, lacosamide, perampanel, zonisamide, topiramate, and pregabalin are good candidates for first-line use. However, because levetiracetam and perampanel can aggravate psychosis, they are frequently replaced with other AEDs when used in NMDAR encephalitis with psychiatric symptoms. Topiramate can cause language problems and memory decline, and its use is sometimes terminated early in NMDAR patients with language dysfunction and memory loss. Patients with aggressive immunotherapy often show neutropenia, which could be a side effect of immunotherapy or a hematologic side effect of AED. In patients being treated with steroid or cyclophosphamide, enzyme-inducing AEDs, such as phenytoin, phenobarbital, and carbamazepine, increase the hepatic degradation of the immunotherapy drugs, dampening the pharmacokinetics. Accordingly, non-enzyme-inducing AEDs are recommended for use in combination with immunotherapeutic agents with hepatic metabolism.

\section{Hypersalivation}

Hypersalivation is one of the symptoms caused by autonomic dysfunction in NMDAR encephalitis with a prevalence of $4 \%$ to $18 \%$ [12]. Hypersalivation is associated with profound drooling, constant suction, pneumonia, and sometimes volume depletion. While anticholinergics are often tried in other diseases, they can aggravate autonomic dysfunction and paralytic ileus in NMDAR encephalitis. Accordingly, effective treatments without systemic side effects are needed.

My team has shown that botulinum toxin injection into the salivary glands can control hypersalivation in NMDAR encephalitis [13]. The effects of one injection last for 12 to 16 weeks and sufficient mitigate the need for other anticholinergics. The injection can be repeated if needed after 12 weeks. Botulinum toxin is a better choice than anticholinergics for management of hypersalivation in NMDAR encephalitis.

\section{Autonomic dysfunction}

Autonomic dysfunction causes fluctuation in blood pressure and heart rate in NMDAR encephalitis. In severe cases, hypotension can cause ischemic organ damage such as ischemic hepatitis. Some patients show sinus arrest, the mechanism for 
which is not fully understood, and need temporary pacemakers. To minimize complications of autonomic dysfunction, anticholinergic drugs should be prescribed with caution. Cholinergic drugs such as pyridostigmine can be helpful, and I frequently prescribe them in patients with paralytic ileus. However, they can increase salivation, and the overall clinical efficacy should be determined in further controlled trials. Midodrine can help to correct hypotension. Blood pressure and heart rate are very sensitive to beta-blockers, and these drugs should be used with caution because they can cause hypotension or bradycardia even at low doses.

\section{Hypoventilation}

Central hypoventilation in NMDAR encephalitis is the main cause of mortality and morbidity via hypoxic brain damage in general wards. If a patient shows rapid progression of the disease, especially altered mentality and dyskinesia, monitoring of oxygen saturation and heart rhythm might be helpful to detect central hypoventilation. Once it occurs, hypoventilation means that the disease course is severe and that 1-year outcomes will be poor, as indicated by the anti-NMDAR Encephalitis One-Year Functional Status (NEOS) score [14]. Intubation, tracheostomy, and ventilator care are inevitable in most patients with central hypoventilation.

\section{Conclusion}

Immunotherapy is the main treatment of NMDAR encephalitis and improves symptoms of the disease. Nevertheless, supportive care is needed to decrease mortality and morbidity. While the protocol for immunotherapy improves every year and several controlled trials on NMDAR encephalitis are being initiated, prospective research is also needed on supportive care topics. Because the final immunologic prognosis of NMDAR encephalitis is usually excellent, the management of organ damage and complications during the disease course is important to prevent permanent physical sequelae of this disease.

\section{Conflicts of Interest}

Soon-Tae Lee has been editorial board of encephalitis since October 2020. He was not involved in the review process of this review article.

Soon-Tae Lee have an advisory role for Genentech, UCB, and Ono Pharmaceuticals outside of the current work, and received research grants from GC Pharma outside of the current work.

\section{References}

1. Lee WJ, Lee ST, Shin YW, et al. Teratoma Removal, Steroid, IVIG, Rituximab and Tocilizumab (T-SIRT) in anti-NMDAR encephalitis. Neurotherapeutics 2020 Sep 3 [Epub]. https://doi.org/10.1007/s13311020-00921-7.

2. Lipton SA. Paradigm shift in neuroprotection by NMDA receptor blockade: memantine and beyond. Nat Rev Drug Discov 2006;5:160170.

3. Mikasova L, De Rossi P, Bouchet D, et al. Disrupted surface cross-talk between NMDA and Ephrin-B2 receptors in anti-NMDA encephalitis. Brain 2012;135(Pt5):1606-1621.

4. Planagumà J, Haselmann H, Mannara F, et al. Ephrin-B2 prevents N-methyl-D-aspartate receptor antibody effects on memory and neuroplasticity. Ann Neurol 2016;80:388-400.

5. Al-Diwani A, Handel A, Townsend L, et al. The psychopathology of NMDAR-antibody encephalitis in adults: a systematic review and phenotypic analysis of individual patient data. Lancet Psychiatry 2019;6:235-246.

6. Lim JA, Lee ST, Kim TJ, et al. Frequent rhabdomyolysis in anti-NMDA receptor encephalitis. J Neuroimmunol 2016;298:178-180.

7. Masdeu JC, Dalmau J, Berman KF. NMDA receptor internalization by autoantibodies: a reversible mechanism underlying psychosis? Trends Neurosci 2016;39:300-310.

8. Shin HR, Jang Y, Shin YW, Chu K, Lee SK, Lee ST. High-dose diazepam controls severe dyskinesia in Anti-NMDA receptor encephalitis. Neurol Clin Pract 2020 Nov 2 [Epub]. https://doi.org/10.1212/CPJ.0000 000000001001 .

9. Kontaxakis VP, Christodoulou GN, Markidis MP, Havaki-Kontaxaki BJ. Treatment of a mild form of neuroleptic malignant syndrome with oral diazepam. Acta Psychiatr Scand 1988;78:396-398.

10. Okoromah CN, Lesi FE. Diazepam for treating tetanus. Cochrane Database Syst Rev 2004;(1):CD003954.

11. Jang Y, Kim DW, Yang KI, et al. Clinical approach to autoimmune epilepsy. J Clin Neurol 2020;16:519-529.

12. Dalmau J, Gleichman AJ, Hughes EG, et al. Anti-NMDA-receptor encephalitis: case series and analysis of the effects of antibodies. Lancet Neurol 2008;7:1091-1098.

13. Jun JS, Seo HG, Lee ST, Chu K, Lee SK. Botulinum toxin treatment for hypersalivation in anti-NMDA receptor encephalitis. Ann Clin Transl Neurol 2017;4:830-834.

14. Balu R, McCracken L, Lancaster E, Graus F, Dalmau J, Titulaer MJ. A score that predicts 1-year functional status in patients with anti-NMDA receptor encephalitis. Neurology 2019;92:e244-e252. 